International Journal of Engineering \& Technology, $7(2.8)(2018) 692-696$
International Journal of Engineering \& Technology
SPC
Website: www.sciencepubco.com/index.php/IJET
Research Paper

\title{
Power quality based optimal nodal pricing in tradable electricity market
}

\author{
Virendra Umale $^{1 *}$, Sanjay Warkad ${ }^{2}$ \\ ${ }^{l}$ Electrical Engineering Department, P.C. Engineering, Nagpur, India \\ ${ }^{2}$ Electrical Engineering Department, P.R.C. Engineering, Amaravti, India \\ *Corresponding Author Email: virendraumale@gmail.com
}

\begin{abstract}
Optimal Power Flow method described the nodal transmission pricing into different related factors, such as congestion, generation, power and electric load limitations. These details of each bus transmission prices can be used for to improve the proper usage of transmission congestion and power grid and to get reasonable transmission pricing for power structure. The proposed methodology is demonstrated on IEEE57 bus system and Maharashtra utility electric 400/765kv network.
\end{abstract}

Keywords: Locational marginal pricing, power quality.

\section{Introduction}

Many developed and developing countries have spent substantial resources and efforts on implementing market-oriented reforms in their electric power sectors since 1990 . Today most of the electricity reform processes are based on the market-orientation approaches to meet variety of objectives i.e. efficiency (techno-economic), competition, privatization, and new regulatory structure [4]. In general, reforms involve the introduction of competition into electricity generation; establishment of regulation; organization of markets for Generation (G), Transmission (T) and Distribution (D); network regulation; privatization of existing assets; promotion of new investments, and allowing for cost-reflective electricity tariffs. The drivers of reforms in developed countries differ from those of developing countries. Enhanced competition and consumer choice have dominated the reform rationale in the former while improved financial and operational performance and increased investment capital for improving electricity service levels are the main driving forces in developing countries. Also the reform programmes adopted by these countries include the elements like introduction of competition into electricity Generation, Transmission, Distribution and supply providers, restructuring the industry in order to enable the introduction of competition, privatization of the unbundled generators and suppliers, development of a new regulatory framework etc. It discover new solution to require load demand at low cost, which satisfied conditions, by adjustment of power system control variables or to find the problem deals with an optimal operating point of a power system that minimizes cost function like generation and transmission loss. This paper design with implementation of electricity pricing in regulated electricity market.

\section{Electricity Spot Pricing}

In regulated markets, pricing required for to solve the bidding issues and get current market values to buyers and sellers. Pricing depends on generator, loads , congestion, private sectors, fuels. Constraints variation gives energy prices from electricity network. The given work is depends on AC-DC power flow transmission pricing. The following modelling for spot pricing is derived for accurate and minimize form for the buyers and sellers. From this pricing sellers and buyers concentrates on actual generation and transmission costing.

\section{Modelling of Spot Pricing}

\section{A. Electricity Nodal Price Equations}

$$
\begin{aligned}
L & =\sum_{i=1}^{N G}\left(a_{i} P_{g i}^{2}+b_{i} P_{G i}+c_{i}\right)+\sum_{i=1}^{N B} \lambda_{p i}\left(P_{d i}-P_{g i}+P_{d c i}+P_{L}\right) \\
& +\sum_{i=N V+1}^{N B} \lambda_{q i}\left(Q_{d i}-Q_{g i}+Q_{d c i}+Q_{L}\right) \\
& +\sum_{i=1}^{N G} \rho p_{l i}\left(P_{g i}^{\min }-P_{g i}\right)+\sum_{i=1}^{N G} \rho p_{u i}\left(P_{g i}-P_{g i}^{\max }\right) \\
& +\sum_{i=1}^{N G} \rho q_{l i}\left(Q_{g i}^{\min }-Q_{g i}\right)+\sum_{i=1}^{N G} \rho q_{u i}\left(Q_{g i}-Q_{g i}^{\max }\right) \\
& +\sum_{i=1}^{N B} \rho v_{l i}\left(\left|V_{i}^{\min }\right|-\left|V_{i}\right|\right)+\sum_{i=1}^{N B} \rho v_{u i}\left(\left|V_{i}\right|-\left|V_{i}^{\max }\right|\right) \\
& +\sum_{i=1}^{N B} \rho \delta_{l i}\left(\delta_{i}^{\min }-\delta_{i}\right)+\sum_{i=1}^{N B} \rho \delta_{u i}\left(\delta_{i}-\delta_{i}^{\max }\right) \\
& +\sum_{i=1}^{N o g l e} \rho p_{f l i}\left(P_{f i}^{\min }-P_{f i}\right)+\sum_{i=1}^{N o g l e} \rho p_{f u i}\left(P_{f i}-P_{f i}^{\max }\right)
\end{aligned}
$$

\section{Problem identify and numerical Results}

\section{A. OPF based Electricity Spot Price: Problem Simulation and Results}

This research methodology has been simulated in Matlab software and results are obtained 
Table 1. AC-DC OPF based Electricity Spot Prices for IEEE57 bus system

\begin{tabular}{|c|c|c|c|c|c|}
\hline Bus no. & voltage & Real power & Reactive power & Angle & $\begin{array}{c}\text { Real spot price } \\
(\$ / \mathrm{Mwh})\end{array}$ \\
\hline 1 & 1.09 & 0.0929 & -0.001 & 0.13 & 20.65 \\
\hline 2 & 1.08 & & 0.4351 & 0.12 & 21.48 \\
\hline 3 & 1.07 & 0.4001 & 0.2991 & 0.06 & 24.21 \\
\hline 4 & 1.07 & & 0.001 & 0.04 & 23.85 \\
\hline 5 & 1.08 & & 0.001 & 0.02 & 23.09 \\
\hline 6 & 1.09 & & 0.501 & 0.02 & 22.57 \\
\hline 7 & 1.07 & & 0.001 & 0.05 & 21.68 \\
\hline 8 & 1.09 & 3.5302 & 0.501 & 0.09 & 20.17 \\
\hline 9 & 1.07 & 0.0001 & 0.501 & 0.04 & 27.16 \\
\hline 10 & 1.07 & & 0.001 & 0.03 & 26.21 \\
\hline 11 & 1.06 & & 0.001 & 0.03 & 26.89 \\
\hline 12 & 1.08 & 4.7001 & 0.501 & 0.07 & 25.08 \\
\hline 13 & 1.06 & & & 0.04 & 26.41 \\
\hline 14 & 1.06 & & & 0.04 & 26.55 \\
\hline 15 & 1.07 & & & 0.06 & 26.37 \\
\hline 16 & 1.08 & & & 0.06 & 24.05 \\
\hline 17 & 1.08 & & & 0.08 & 22.54 \\
\hline 18 & 1.06 & & & -0.02 & 24.05 \\
\hline 19 & 1.01 & & & -0.03 & 25.69 \\
\hline 20 & 1 & & & -0.02 & 26.28 \\
\hline 21 & 1.02 & & & -0.02 & 27.02 \\
\hline 22 & 1.02 & & & -0.01 & 27.08 \\
\hline 23 & 1.02 & & & -0.01 & 27.08 \\
\hline 24 & 1.02 & & & -0.01 & 26.38 \\
\hline 25 & 0.97 & & & -0.1 & 26.96 \\
\hline 26 & 0.98 & & & -0.01 & 26.18 \\
\hline 27 & 1.03 & & & 0.01 & 23.99 \\
\hline 28 & 1.06 & & & 0.02 & 22.96 \\
\hline 29 & 1.08 & & & 0.03 & 22.22 \\
\hline 30 & 0.95 & & & -0.11 & 27.75 \\
\hline 31 & 0.93 & & & -0.12 & 28.93 \\
\hline 32 & 0.95 & & & -0.11 & 28.55 \\
\hline 33 & 0.95 & & & -0.11 & 28.65 \\
\hline 34 & 0.97 & & & -0.03 & 28.73 \\
\hline 35 & 0.98 & & & -0.03 & 28.51 \\
\hline 36 & 0.99 & & & -0.03 & 28.16 \\
\hline 37 & 0.99 & & & -0.02 & 27.88 \\
\hline 38 & 1.02 & & & -0.01 & 27.11 \\
\hline 39 & 0.99 & & & -0.02 & 27.95 \\
\hline 40 & 0.98 & & & -0.03 & 28.21 \\
\hline 41 & 1.03 & & & -0.03 & 26.77 \\
\hline 42 & 0.99 & & & -0.05 & 28.04 \\
\hline 43 & 1.05 & & & 0.01 & 26.86 \\
\hline 44 & 1.03 & & & 0 & 26.91 \\
\hline 45 & 1.06 & & & 0.02 & 26.12 \\
\hline 46 & 1.04 & & & 0.01 & 26.44 \\
\hline 47 & 1.03 & & & 0 & 26.84 \\
\hline 48 & 1.02 & & & 0 & 26.92 \\
\hline 49 & 1.03 & & & 0 & 26.72 \\
\hline 50 & 1.02 & & & -0.01 & 27.09 \\
\hline 51 & 1.06 & & & 0.01 & 26.16 \\
\hline 52 & 1.05 & & & 0.08 & 23.44 \\
\hline 53 & 1.04 & & & 0.11 & 23.81 \\
\hline 54 & 1.08 & & & 0.21 & 22.37 \\
\hline 55 & 1.04 & & & 0.08 & 26.46 \\
\hline 56 & 0.98 & & & -0.06 & 28.46 \\
\hline 57 & 0.97 & & & -0.07 & 28.74 \\
\hline
\end{tabular}

Table 2. AC-DC OPF for IEEE57 bus system with different voltage limitations

\begin{tabular}{|c|c|c|c|}
\hline Bus no & $\begin{array}{c}\text { Nodal price } \\
\text { dollar/kwh }\end{array}$ & $\begin{array}{c}\text { Nodal price } \\
\text { dollar/kwh }\end{array}$ & Nodal price dollar/kwh \\
\hline 1 & 20.65 & 20.45 & 20.30 \\
\hline 2 & 21.48 & 21.28 & 21.13 \\
\hline 3 & 24.21 & 24.01 & 23.86 \\
\hline 4 & 23.85 & 23.65 & 23.50 \\
\hline 5 & 23.09 & 22.89 & 22.74 \\
\hline 6 & 22.57 & 22.37 & 22.22 \\
\hline 7 & 21.68 & 21.48 & 21.33 \\
\hline 8 & 20.17 & 19.97 & 19.82 \\
\hline 9 & 27.16 & 26.96 & 26.81 \\
\hline 10 & 26.21 & 26.01 & 25.86 \\
\hline
\end{tabular}




\begin{tabular}{|c|c|c|c|}
\hline 11 & 26.89 & 26.69 & 26.54 \\
\hline 12 & 25.08 & 24.88 & 24.73 \\
\hline 13 & 26.41 & 26.21 & 26.06 \\
\hline 14 & 26.55 & 26.35 & 26.20 \\
\hline 15 & 26.37 & 26.17 & 26.02 \\
\hline 16 & 24.05 & 23.85 & 23.70 \\
\hline 17 & 22.54 & 22.34 & 22.19 \\
\hline 18 & 24.05 & 23.85 & 23.70 \\
\hline 19 & 25.69 & 25.49 & 25.34 \\
\hline 20 & 26.28 & 26.08 & 25.93 \\
\hline 21 & 27.02 & 26.82 & 26.67 \\
\hline 22 & 27.08 & 26.88 & 26.73 \\
\hline 23 & 27.08 & 26.88 & 26.73 \\
\hline 24 & 26.38 & 26.18 & 26.03 \\
\hline 25 & 26.96 & 26.76 & 26.61 \\
\hline 26 & 26.18 & 25.98 & 25.83 \\
\hline 27 & 23.99 & 23.79 & 23.64 \\
\hline 28 & 22.96 & 22.76 & 22.61 \\
\hline 29 & 22.22 & 22.02 & 21.87 \\
\hline 30 & 27.75 & 27.55 & 27.40 \\
\hline 31 & 28.93 & 28.73 & 28.58 \\
\hline 32 & 28.55 & 28.35 & 28.20 \\
\hline 33 & 28.65 & 28.45 & 28.30 \\
\hline 34 & 28.73 & 28.53 & 28.38 \\
\hline 35 & 28.51 & 28.31 & 28.16 \\
\hline 36 & 28.16 & 27.96 & 27.81 \\
\hline 37 & 27.88 & 27.68 & 27.53 \\
\hline 38 & 27.11 & 26.91 & 26.76 \\
\hline 39 & 27.95 & 27.75 & 27.60 \\
\hline 40 & 28.21 & 28.01 & 27.86 \\
\hline 41 & 26.77 & 26.57 & 26.42 \\
\hline 42 & 28.04 & 27.84 & 27.69 \\
\hline 43 & 26.86 & 26.66 & 26.51 \\
\hline 44 & 26.91 & 26.71 & 26.56 \\
\hline 45 & 26.12 & 25.92 & 25.77 \\
\hline 46 & 26.44 & 26.24 & 26.09 \\
\hline 47 & 26.84 & 26.64 & 26.49 \\
\hline 48 & 26.92 & 26.72 & 26.57 \\
\hline 49 & 26.72 & 26.52 & 26.37 \\
\hline 50 & 27.09 & 26.89 & 26.74 \\
\hline 51 & 26.16 & 25.96 & 25.81 \\
\hline 52 & 23.44 & 23.24 & 23.09 \\
\hline 53 & 23.81 & 23.61 & 23.46 \\
\hline 54 & 22.37 & 22.17 & 22.02 \\
\hline 55 & 26.46 & 26.26 & 26.11 \\
\hline 56 & 28.46 & 28.26 & 28.11 \\
\hline 57 & 28.74 & 28.54 & 28.39 \\
\hline
\end{tabular}

Graph 1. AC-DC OPF for IEEE57 bus system with different voltage limitations

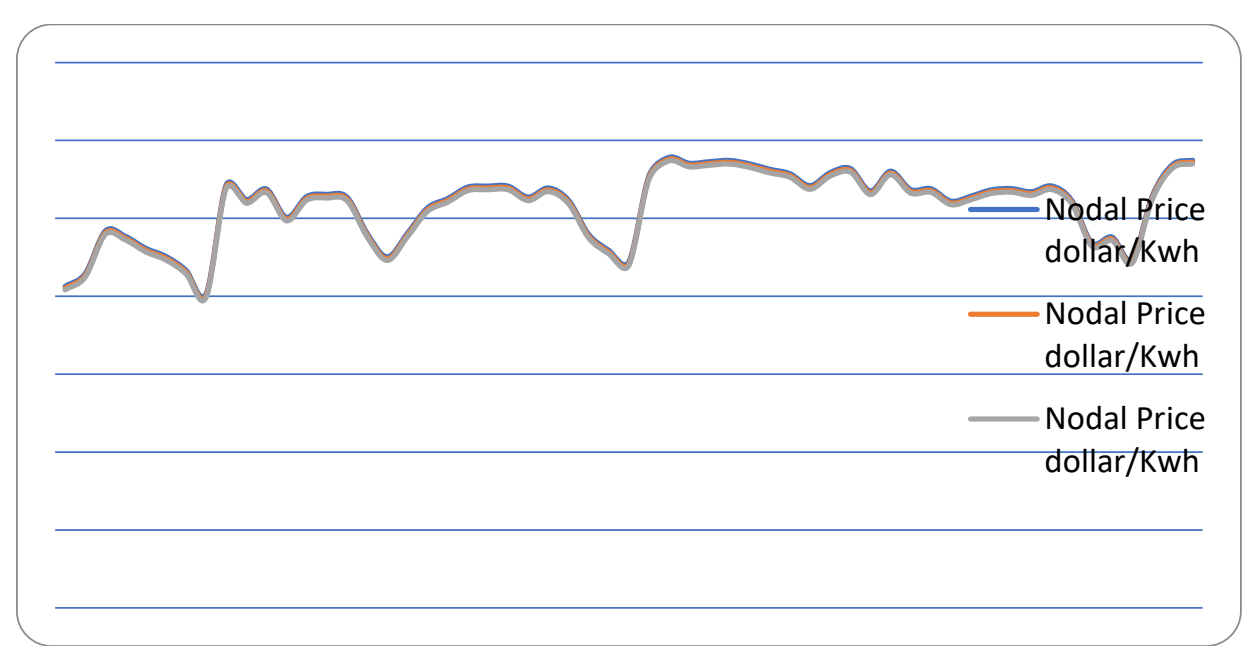


Table 3. OPF based electricity spot transmission pricing using for Maharashtra Network Electricity Network

\begin{tabular}{|c|c|c|c|c|c|}
\hline Bus No. & Voltage & Real power & $\begin{array}{c}\text { Reactive } \\
\text { power }\end{array}$ & Angle & $\begin{array}{l}\text { Spot price } \\
\text { (Rs./kwh) }\end{array}$ \\
\hline 1 & 418.39 & 2.59 & 1.97 & 0.15 & 1.88 \\
\hline 2 & 413.66 & 2.66 & 0.54 & 0.27 & 1.87 \\
\hline 3 & 420.01 & 2.54 & & & 1.91 \\
\hline 4 & 412.57 & 2.5 & & & 1.93 \\
\hline 5 & 416.72 & 2.55 & & & 1.92 \\
\hline 6 & 417.92 & 2.66 & & & 1.89 \\
\hline 7 & 400.73 & 1.85 & & & 2.24 \\
\hline 8 & 394.13 & 1.84 & & & 2.29 \\
\hline 9 & 393.7 & 1.83 & & & 2.29 \\
\hline 10 & 413.44 & 1.88 & & & 2.13 \\
\hline 11 & 409.4 & 1.86 & & & 2.16 \\
\hline 12 & 415.08 & 1.94 & 0.8 & 0.13 & 2.14 \\
\hline 13 & 414.34 & 1.93 & & & 2.14 \\
\hline 14 & 413.73 & 1.93 & 0.1 & 0.18 & 2.14 \\
\hline 15 & 394.61 & 1.89 & & & 2.31 \\
\hline 16 & 420.01 & 1.82 & & & 2.14 \\
\hline 17 & 409.15 & 1.94 & & & 2.13 \\
\hline 18 & 406.22 & 2.09 & & & 2.07 \\
\hline 19 & 408.76 & 2.19 & & & 2.03 \\
\hline 20 & 389.82 & 2.6 & 0.4 & 0.41 & 1.92 \\
\hline 21 & 400.7 & 2.57 & & & 1.92 \\
\hline 22 & 403.76 & 2.54 & & & 1.92 \\
\hline 23 & 420.01 & 2.38 & & & 1.97 \\
\hline 24 & 418.59 & 2.34 & & & 1.98 \\
\hline 25 & 420.01 & 2.6 & 0.4 & 0.12 & 1.86 \\
\hline 26 & 420.01 & 2.92 & 0.51 & 0.03 & 1.78 \\
\hline 27 & 389.25 & 2.55 & 0.11 & 0.6 & 1.92 \\
\hline 28 & 420.01 & 3.01 & 0.85 & 0.14 & 1.79 \\
\hline 29 & 409.13 & 2.69 & 0.1 & 0.25 & 1.89 \\
\hline 30 & 401.55 & 1.91 & 0.2 & 0.01 & 2.22 \\
\hline 31 & 401.38 & 1.87 & 0.2 & 0.01 & 2.24 \\
\hline
\end{tabular}

Table 4. AC-DC OPF based Electricity Spot Prices for 400/765kv MSTECL different voltage limitations

\begin{tabular}{|c|c|c|c|}
\hline Bus no. & $\begin{array}{l}\text { Nodal price } \\
\text { Rs/kwh }\end{array}$ & $\begin{array}{l}\text { Nodal price } \\
\text { Rs/kwh }\end{array}$ & Nodal price Rs/kwh \\
\hline 1 & 1.88 & 1.76 & 1.63 \\
\hline 2 & 1.87 & 1.75 & 1.62 \\
\hline 3 & 1.91 & 1.79 & 1.66 \\
\hline 4 & 1.93 & 1.81 & 1.68 \\
\hline 5 & 1.92 & 1.8 & 1.67 \\
\hline 6 & 1.89 & 1.77 & 1.64 \\
\hline 7 & 2.24 & 2.12 & 1.99 \\
\hline 8 & 2.29 & 2.17 & 2.04 \\
\hline 9 & 2.29 & 2.17 & 2.04 \\
\hline 10 & 2.13 & 2.01 & 1.88 \\
\hline 11 & 2.16 & 2.04 & 1.91 \\
\hline 12 & 2.14 & 2.02 & 1.89 \\
\hline 13 & 2.14 & 2.02 & 1.89 \\
\hline 14 & 2.14 & 2.02 & 1.89 \\
\hline 15 & 2.31 & 2.19 & 2.06 \\
\hline 16 & 2.14 & 2.02 & 1.89 \\
\hline 17 & 2.13 & 2.01 & 1.88 \\
\hline 18 & 2.07 & 1.95 & 1.82 \\
\hline 19 & 2.03 & 1.91 & 1.78 \\
\hline 20 & 1.92 & 1.8 & 1.67 \\
\hline 21 & 1.92 & 1.8 & 1.67 \\
\hline 22 & 1.92 & 1.8 & 1.67 \\
\hline 23 & 1.97 & 1.85 & 1.72 \\
\hline 24 & 1.98 & 1.86 & 1.73 \\
\hline 25 & 1.86 & 1.74 & 1.61 \\
\hline 26 & 1.78 & 1.66 & 1.53 \\
\hline 27 & 1.92 & 1.8 & 1.67 \\
\hline 28 & 1.79 & 1.67 & 1.54 \\
\hline 29 & 1.89 & 1.77 & 1.64 \\
\hline 30 & 2.22 & 2.1 & 1.97 \\
\hline 31 & 2.24 & 2.12 & 1.99 \\
\hline
\end{tabular}


Graph 2. OPF based Electricity Spot Prices for 400/765kV MSETCL with different voltage limitations

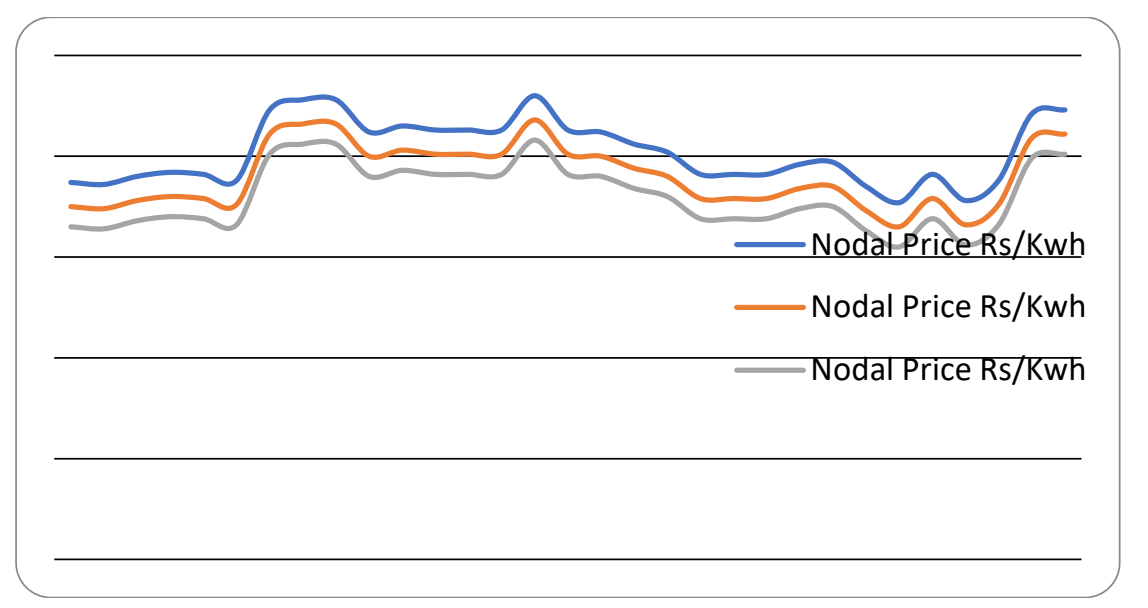

\section{Conclusion}

The paper presented power quality based optimal electricity pricing methodology suitable for Indian real power systems. For power quality, changes the different voltage values and according the nodal prices are get suitable transmission pricing. The power quality improves the system as well as grid with proper voltages. For better quality assumed different load conditions, generation and transmission congestion.

\section{References}

[1] contreras, J., Espínola, R., Nogales, F. J., and Conejo, A. J. ARIMA models to predict nextday electricity prices.

[2] onejo, A. J., Plazas, M. A., Espinola, R. and Molina, A. B. Dayahead electricity price forecasting using the wavelet transform and ARIMA models. IEEE Transactions on Power System,20 (2), 2005, 10351042.

[3] Garcia, R. Contreras, J., Akkeren, M. and Garcia, J. B. C. A GARCH forecasting model to predict day ahead electricity prices. IEEE Transactions on Power System, 20 (2), 2005, 867-874.

[4] Fan, S., Mao, C. and Chen L. Next-day electricity-price forecasting using ahybrid network. IET Generation Transmission Distribution, 1 (1), 2007, 176-182.

[5] Li, Guang, Liu, Ching,Mattson, Chris and Lawarrée, J. Day-Ahead Electricity Price Forecasting in a Grid Environment. IEEE Transactions on PowerSystem,22 (1), 2007, 266-274.

[6] Pavlos S., Georgilakis Artificial Intelligence solution to electricity price forecasting problem. Journal of Applied Artificial Intelligence, 21 (8), 2007, 707-727.

[7] Rodriguez, C. P. and Anders, G. J. Energy price forecasting in the Ontario competitive power system market. IEEE Transactions. on Power System, 19(1), 2004, 366-374.

[8] Zhang, L. and Luh, P. B. Neural network-based market clearing price prediction and confidence interval estimation with an improved extended Kalman filter method. IEEE Transactions on Power System,20 (1), 2005

[9] Mandal, P., Srivastava, A.K., and Park, Jung Wook. An Effort to Optimize Similar Days Parameters for ANN Based Electricity Price Forecasting. IEEE Transactions on Industry Applications, 45 (5), 2009

[10] S.b.Warkad ,M.K.Khedkar,AC-DC OPF based Day-Ahead Electricity Nodal Price Prediction using an ANN I.J.C.A Vol.9N0.10,Nov.2010

[11] G. Ward, N. R. Watson, C. P. Arnold, A. J. Turner, and B. J. Ring, "Inversion of Real Time Spot Prices in the Direction of Real Power Flow in a Transmission Line", IEEE Transactions on Power Systems, vol.15, no.4, pp. 1197-1203, November 2000.

[12] Luonan Chen, Hideki Suzuki, Tsunehisa Wachi, and Yukihiro Shimura, "Components of Spot Prices for Electric Power Systems", IEEE Transactions on Power Systems, vol. 17, no.1, pp.41-49, February 2002

[13] S.B.Warkad, Dr. M.K. Khedkar, Dr. G. M. Dhole, "Optimal Electricity Nodal Pricing in a Restructured Electricity Market", Journal of The Institution of Engineers (India), Volume 91, pp. 2935 , June $18,2010$.
[14] S.V.Manikanthan and K.Baskaran "Low Cost VLSI Design Implementation of Sorting Network for ACSFD in Wireless Sensor Network", CiiT International Journal of Programmable Device Circuits and Systems,Print: ISSN 0974 - 973X \& Online: ISSN 0974 - 9624, Issue : November 2011, PDCS112011008.

[15] T. Padmapriya and V. Saminadan, "Improving Throughput for Downlink Multi user MIMO-LTE Advanced Networks using SINR approximation and Hierarchical CSI feedback", International Journal of Mobile Design Network and Innovation- Inderscience Publisher, ISSN : 1744-2850 vol. 6, no.1, pp. 14-23, May 2015. 